\title{
NEW INTUITION ON EAR AUTHENTICATION WITH GABOR FILTER USING FUZZY VAULT
}

\author{
A. Kavipriya \\ Department of Electronics and Communication Engineering, \\ Kalasalingam Academy of Research and Education, \\ Krishnankoil, (India).
}

E-mail: kavidivya222@gmail.com ORCID: https://orcid.org/0000-0002-2965-2542

M. Arunachalam

Department of Electronics and Communication Engineering,

Kalasalingam Academy of Research and Education,

Krishnankoil, (India).

E-mail: muthuece.eng@gmail.com ORCID: https://orcid.org/0000-0001-8070-3475

\section{Citación sugerida:}

Kavipriya, A., y Arunachalam, M. (2020). New Intuition on Ear Authentication with Gabor Filter Using Fuzzy Vault. 3C Tecnología. Glosas de innovación aplicadas a la pyme. Edición Especial, Marzo 2020, 159-179. http://doi.org/10.17993/3ctecno.2020.specialissue4.159-179

\section{Suggested citation:}

Kavipriya, A., \& Arunachalam, M. (2020). New Intuition on Ear Authentication with Gabor Filter Using Fuzzy Vault. 3C Tecnología. Glosas de innovación aplicadas a la pyme. Edición Especial, Marzo 2020, 159-179. http://doi.org/10.17993/3ctecno.2020.specialissue4.159-179 


\section{ABSTRACT}

At present, Frequent Biometrics Scientific Research deals with other biometric application like Face, Iris, Voice, Hand-Based Biometrics traits for classification and spotting out the persons. These Specific Biometric traits have their own improvement and weakness for opting the terms like Accuracy \& cost of all applications. However, in addition to other Face-based Biometric techniques, Ear Recognition has been appealed to Boom the attention among other Biometric researchers. This Image Template Pattern Formation of Ear cuddles the report which is relevant for maculating the Uniqueness of their individuality. This Ear Biometric trait observes the person's identity based on its stable Anatomical behavior. This biometric trait does not involve any emotional feelings with facial expressions in the same way as a unique pair of Fingerprint. In this work, a Contemporary approach for Personal identification is imported with Ear along with the data stores in a secured way has been proposed. This authentication Process includes the revolution of features with Gabor Filter and Dimension Reduction based on Multi-Manifold Discriminant Analysis (MMDA). This work is adequately analyzed in Matlab with the Evaluation metrics such as FMR, GAR, FNMR, by modifying the key value each time. The results of this suggested work promote better values in recognition of individuals as for Ear modalities. Conclusively the Features are grouped using K-Means for both identification and Verification Process. This Proposed system is initialized with Ear Recognition Template based on Fuzzy Vault. The Key stored in the Fuzzy Vault is utilized in safeguarding the existence of Chaff Points.

\section{KEYWORDS}

2D Gabor Filter, Multi-Manifold Discriminant Analysis (MMDA), K-Means, False Matching Rate (FMR), False Non Matching Rate (FNMR), Genuine Acceptance Rate (GAR). 


\section{INTRODUCTION}

In the present day scenario, the booming demand in case for both Security and automated recognition system leads to radical research resolution in the various areas of Computer Vision and Intelligent systems. At Present Periodic arrangements of individual identity happens through the enactment of Password with Permissive Activities in Public security, Access Control, Computer Vision as well as Intelligent Systems. Therefore Biometrics is considered as a significant application of forensics, Surveillance examination which assigns to the technique of diagnosing the Humans by utilizing their physical or behavioral traits along with faces, Iris, Fingerprint, Ear, Palm print, FKP, voice, and signature. These features can be treated as Biometric diagnostic features with satisfaction of requirements: (i)Universality, (ii)Distinctiveness, (iii)Permanence, (iv)Performance, (v)Collectability, (vi) Acceptability. Each of these above mentioned biometric procedure has both its precedence and nuisance using single modality which is optimal for other types of Professional systems applications. This paper targets on Human Ear as one of the auspicious and idiosyncratic biometric modality that involves enduring and dependent with a shape which does not expose desperate contradiction with age.

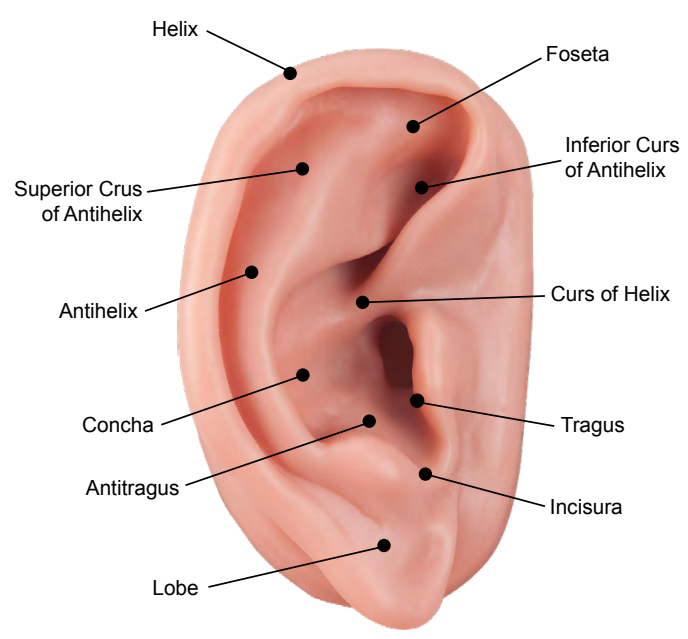

Figure 1. Anatomy of Human Ear.

Based on Figure 1 explains the External anatomical Structure of Human Ear with its lingual components including Helix, Antihelix, Tagus, Concha, Lobe and further Parts. This Infinite ridges and Valleys on the external Ear's Surface act as phonic Signals. In case 
of Low Frequencies, Pinna reproduces the acoustic signals near to the ear canal. Similarly, High Frequencies reverberate the acoustic wave which causes the frequencies to be calm down. This makes to note that origin of Sound perceived enabled by humans in Outer Ear. Random Factors of Ear's appearances can be first examined by correlating the Left and Right Ear of the similar Persons.

The ear Recognition can be approximately restricted based on the categories of several methods like Hassaballah, Alshazly, and Ali (2019) explains the EAR authentication complication utilizing Local Binary Patterns (LBP) Features. This Monotonic image of Gray-Scale factor transforms with its computational efficiency with Local Binary Pattern features that is fit for Ear Recognition problem. This tested LBP variants almost show the accuracy rate around 99\%, while the attainment faces several difficulties when the level of distortion boost. Likewise, Gandhimathi and Janarthanan (2019) describe the new class of Biometric as Ear recognition in comparison of Fingerprint that can be smoothly confiscated from the area's measures. Alike due to the emotion the shape of Ear does not change even due to the emotion. It is relatively constant over a Person's life. Robust Feature extraction helps in determining the personality of several individuals, for instance, terrorists at airports \& stations. Similarly, Gandhimathi and Radhamani (2016), and Gandhimathi and Janarthanan (2019), defines the effective fusion method for the combination of various data that can be secured by the generation of chaff points. These chaff points help in the formation of a secret key using the unimodal biometric data with feature vectors. The optimal location of these feature vectors is basically created by the fitness value as well as the development of security enhancement with the help of multi-biometric systems by means of the proposed modified template of Log Gabor Features XOR pattern. This kind of template security is basically determined by the other way of fuzzy vault multi- biometric cryptosystem. Vinothkanna and Amitabh (2014) explains the grouped feature vector resemblance points that are developed by chaff points and feature points. This grouped vector points in the fuzzy vault database lead to the accurate identification of the recognized individuals with correct match points. Several evaluation metrics like FAR, FRR, GAR, Secret key helps in the assessment of grouped vector points. Bansal and Hanmandlu (2017) presents the ear based identification function by the means of entropy values with reference to change in information gain information score values. This effective 
Gaussian and Exponential function find in generating the refined scores that are facilitated using the Euclidean distance metrics. Anwar, Ghany, and Elmahdy (2015) developed an advanced algorithm in case of Ear Recognition with geometric features extraction. Here Ear recognition with geometric features extraction. The ear detection generally based on the snake model with a median filter for removal of noises. Then canny edge and distance metrics are created with these image features. This method is invariant to translation\& rotation with accuracy of 98\%. Kacar and Kirci (2018) introduced the novel architecture with Score Net in case of Ear recognition. This method creates with modality pool in accordance with cascade fusion learning that is compatible with parallel processing. Lakshman (2013) implemented the double-stage geometric approach in both scale features $\&$ rotation invariant in case of uprooting the unique features. Hence the matching scores are compared with the basics of threshold values with authenticating the persons. Herewith PSO technique helps in Optimize the parameters with threshold and weights which helps in regulating the computation time. Rathgeb, Pflug, Wagner, and Busch (2016) deals with the image compression that helps in ear recognition stages with stimulated image distortions and partitions. Finally the detailed investigation of image compression technique. The feature extraction was calculated with uncompressed samples of Ear databases with numerous bitrates. Pflug and Busch (2012) discovered the identification by Ear recognition in case of 2D, 3D images in case of smart surveillance \& forensic image analysis. It explains the database collection with various features against various techniques. Nandakumar, Jain, and Pankanti (2007) extracts the highest curvature points that are helpful in aligning the template. Minutiae Matcher of decoding part leads to non-linear distortion which gives a significant improvement of GAR. Koptyra and Ogiela (2015) present a unique idea of hiding the secrets using the fuzzy vault. It is mainly hidden the noisy data based on multibiometric cryptosystem. It proposes a choice of authentication accuracy relevant with a cryptosystem on single biometric. Bae, Noh, and Kim (2003) shows the encoding of iris code that helps in the performance of EER that gives the magnitude performance for iris size along with processing time. Arunachalam and Kanan (2015) integrate the secret key value using Advanced Encryption Standard to avoid several attacks like spoofing, intraclass variations, etc., for the generation of biometric key utilizing the cryptographic fusion Uludag and Jain (2006) aims in the safeguarding and aloofness of biometric systems with the 
transformed version of the template that is stored as a cryptographic framework. So they introduce the orientation field of helper data for the extraction of fingerprints. Yang, Sun, and Zhang (2011) proposed the dimensionality reduction method for pattern recognition purposes that is based on graph embedded learning. This technique mainly based on the construction of low dimensional data. Basically, it cannot apply for small size problem. To overcome this, MMDA is calculated for Eigenvectors and Eigen value representations. Yang, Sun, and Wang (2011) have attracted interest against Gabor feature with MMDA. Certain Fuzzy vault system is generally on the support of local iris feature points from the exact values of an unordered set with basis if shift matching technique.

Remaining paper is formulated as follows: Section $2 \& 3$ precedes the inquiry of scheduled work and it portrayed the Fuzzy Vault which includes eradication of Ear along with Gabor features and grouped according to k-Means clustering algorithm in a detailed manner and Section 4 provides the particulars in relation to the basic Fuzzy Vault construction with enrolment and verification phase. Experimental decision is essentially explained in Section 5 and finally $6^{\text {th }}$ section entirely organizes the basic work that provides points for future research. The motivation of this work involves the Human Authentication that must be considered as the most important tasks which are used in this world for the case of identification of Persons using biometrics with its Physical and Behavioral Characteristics. They include Fingerprints, Handprints, Palm prints, Hand veins, Eyes, Ears, Voice, and signature. Basically, this biometric system is categorized as Unimodal; Multimodal, and Multi-biometric system, etc. This unimodal biometric System has severe challenges against noisy data. In this work, the Ear modalities are selected to generate the polynomial construction to the let the secure key in a collapsed manner. The reason for selecting the ear as main modalities is due to surprising rich features with it. Changes do not happen due to its stable structure.

\section{SYNOPSIS OF THE PROPOSED WORK}

This proposed paper suggested the flow diagram that explains briefly about the Ear recognition with the generation of polynomial construction in both locking and unlocking set for the case of Fuzzy Vault system. This work illustrates the cryptographic fuzzy vault technique as three phases. In Recognition phase the Ear images are collected from the 
database for the further process of dimensionality reduction phase. Further the enrollment phase the Gabor feature extraction is carried with five scales and eight orientations. These extracted features basically have high dimensional values. Minimization of this idea is accessed by the MMDA Technique in the calculation of Eigen value and Eigenvector that are explained below in this block Diagram.

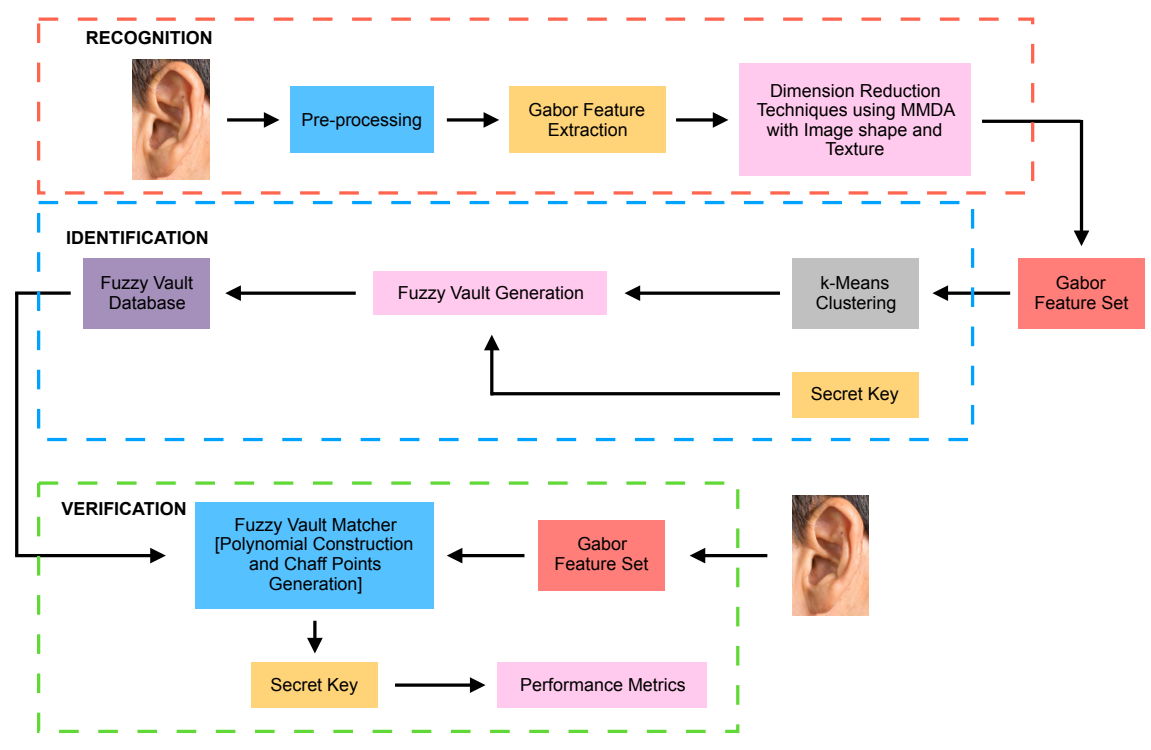

Figure 2. Flow diagram of proposed work with Fuzzy Vault Technique.

This Figure 2 shows the process of Ear recognition with several methods that include based on Fuzzy Vault. This vault helps in providing the security to several biometric cryptosystems. Here the chaff points are formed promptly from the biometric features which are identified easily. The features are clustered based on manifold learning Process. The origination of chaff points or secret key by the process of the vault locking process. Locking process creates the Polynomial generation of chaff points as a key that must be entered. Similarly, the Testing phase the same procedure is repeated in order to assess the common features and matching is done based on the revealing of the secret key along with biometrics. There are four significant stages in this proposed work:

\section{A. Pre-processing.}

\section{B. Gabor Feature Extraction.}




\section{Generation of Polynomial construction of grouped feature vectors.}

\section{Identification and Authentication of Secret Key.}

\section{A. Pre-Processing Phase}

Since the early phase, the images are to be pre-processed along with the objective of getting rid of the rejected part in image such as noise, Blur, reflections. Originally, these Ear images are reformed into gray scale images in the datasets are in RGB format. Thus the training process is enforced with ear datasets. Specific basic non-linear methods recycled are the median Filter. The main method of this filter helps in glaring of edges that helps in reducing the noises with the point of subsiding the current pixel point with the median of illumination in its range.

This center pixel appraisal is named as "median" and similarly the neighboring pattern as "window".

$$
H(m, n)=\operatorname{median}[x(m-k, n-l) \hat{I} w]
$$

This equation 1 " $w$ " imitates the window along with the pixels $(m, n)$. Here the inured input images of the ear are expertly pre-processed and represented as $I_{e}$. Further this images separable which is cropped out to obtain the ROI with the help of changing the image size and Pixels.

\section{B. Gabor Feature Extraction\& k-means clustering}

Gabor feature Extraction is based on spatial locality and oriented selectivity with Ear Images. Gabor wavelets formation is developed. Gabor wavelet formation is developed with the kernels which are to identical to certain profiles and exposing the desirable location and orientation selectivity. This Gabor wavelet determination is to be entitled as:

$$
\left.\psi_{u, v(z)}=\left\|\frac{k_{u, v}}{\sigma_{2}}\right\|^{2} e^{\left(-\left|\frac{k(u, v)}{2 \sigma^{2}}\right|^{z}\right.}\right)\left(e^{i k_{u, v}} e^{\frac{-\sigma^{2}}{2}}\right)
$$

Where $\mathrm{u}, \mathrm{v}$ denotes the direction, scales of Gabor feature kernels. It is defined based on norm operator 
Where:

$$
k_{u, v}=\frac{k \max }{\frac{v}{2}}
$$

This factor "K max" represents high frequency and $\mathrm{f}$ depicts spacing vector with five scales and eight orientations. Further convolution of Gabor features is based on $Z(x, y)$ that serves the ultimate position of the figure and *denotes convolution operator.

\section{Multi-Manifold Discriminant Analysis (MMDA)}

Collection of Sample set with various ear data label is denoted as

$$
A=\left[A_{1} A_{2}, A_{3} \ldots A_{N}\right] A_{i} \sum R_{M}
$$

Likewise the linear projection of low dimensional space is defined as the

$$
B=P^{T} A^{r}
$$

Considering the points with several similar class labels that Possess edge construction between the nodes $y_{i}, y_{j}$ from the corresponding class. It is also broadly promoted such as $y_{i}, y_{j}$ with its parameter

$$
o \leq s_{i j} \leq 1
$$

Here weight functions are taken as an important note with strict monotonically decreasing function. Apparently, it has been noticed with negative non-symmetric that are exalted by the matrices between Class and within class scatter in $\beta_{W}, \beta_{B}$

$$
J(P)=\arg _{p} \max \equiv \frac{J_{R}(P)}{J_{W}(P)}
$$

Therefore it can be represented as:

$$
\frac{P^{T} B_{b} P}{P^{T} B_{W} P}
$$


Hence the projection matrix is generally represented as:

$$
\beta_{b} p=\lambda \beta_{w} p
$$

This projection matrix is literally named after the Graph embedding algorithm which is intended by the Eigen Value. These processes are clustered by the part of k-means clustering by calculating the centroid points and accredited these points towards the center.

\section{Glustering using K-means}

Clustering mainly used to acclimate the feature points based on the performance of unsupervised classification of certain patterns as groups. Considering the size of input and classification in large groups, $\mathrm{k}$-means clustering target the execution process with a basis of Ear feature points. Further, it is continued based on centroid calculation. Basically, it is like the expectation-maximization algorithm with mixtures of Gaussian in the process of finding clustering with various attributes.

$$
J=\sum_{j=1}^{k} \sum_{i=1}^{n}\left\|x_{i}^{j}-c_{1}^{2}\right\|
$$

Where J represents the objective function that is to be defined number of cases and centroid for cluster points that are based on Euclidean distance with distance measure defined as the classification of objects.

\section{Algorithm:}

Input: $\mathrm{k}$ and other points with $\mathrm{b}_{1}, \mathrm{~b} 2 \ldots \ldots \mathrm{b}$; Clustering the data into several $\mathrm{k}$ groups.

Gluster Update: Selecting k points at random cluster Centers.

Centers Update: Assigning articles to the adjoining cluster Centre to determine according to the Euclidean distance.

Stopping Update: Determining the centroid points or mean of severalEar featuresin ever Cluster.

Output: Repetition of steps 2, 3 until similar points assigned to each cluster. 


\section{c. Generation of Polynomial construction of grouped feature vectors}

In order to assigning the template security, Secret key plays the main role in generating the fuzzy vault that is united to form grouped feature vector. Originally the intake of secret keyis concealed with the number of chaff point's generation. Considering the information stored in the dataset is Permanent, Security is taken as an important note. Fuzzy vault is radically a cryptographic construction recommended by Juels and Sudan (2006) securing the critical data with the help of biometric data.

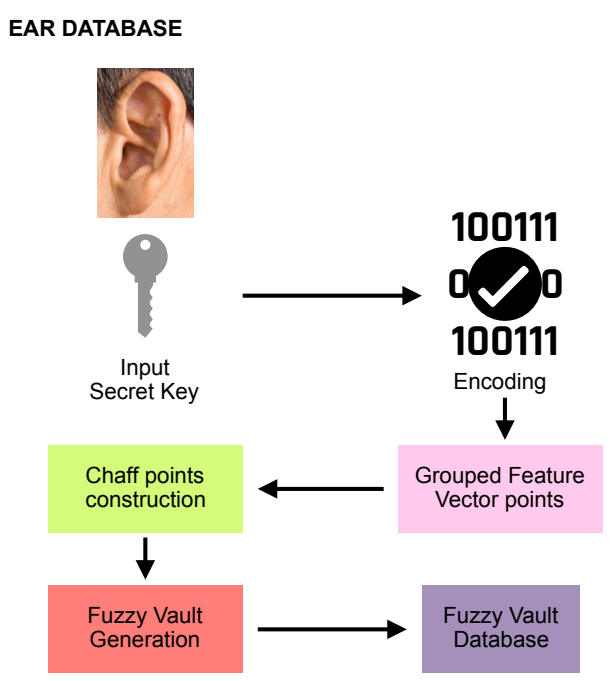

Figure 3. Block diagram of Fuzzy vault Construct.

Based on Figure 3 Polynomial construction with genuine points are stored as a secret key from the Ear database. Usually, the secret key information that is distributed as unordered sets named as Chaff points. These chaff points basically denote the content of secure information to be reconstructed for revealing the secret code which is stored in the Fuzzy vault database.

\section{d. Identification and Authentication of Secret Key}

In the recognition phase, Person's ear images are taken as input that is pre-processed and the features are extracted for the combination of feature vector. This input feature vector is compared to the fuzzy vault database. Matching relates with the secret key generation and authentication is proved. This recognition process is adorned. Let the given person's 
Gabor feature vector that must express by $c$ that is related to the fuzzy vault in the dataset. In case if every feature points of the ear image matches the features in the fuzzy vault, then the individual is admitted authentication or else the authentication is contradicted. Assured points in the fuzzy vault will be left deserted. These points are named as "secret points" and the x-coordinates of these features' points provide the secret key of the authenticated person.

Finally the procreation of the authenticated person is the second confirmation of the person which boosts the template security.

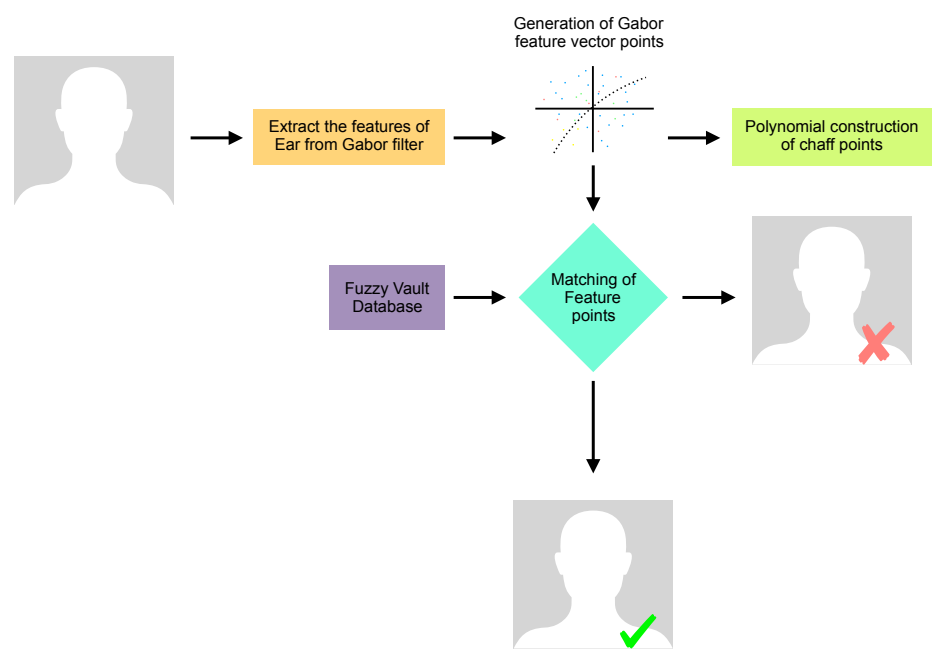

Figure 4. Recognition of a person with Fuzzy vault using Ear.

This Figure 4 represents the recognition of the person based on the person's ear image with Fuzzy vault. These features which are extracted from the Gabor filters are generated to form the polynomial construction of chaff points. These matched feature points are determined from the vault database gives the authentication of the person.

\section{EXPERIMENTAL RESULTS AND PERFORMANCE EVALUATION}

In this category, the consequences of the designed biometric method for the recognition of Ear modalities utilizing Fuzzy Vault are contended with detailed manner in this work. This Intended methodology is executed in Matlab Platform of version 2017. Dataset confession of IIT Delhi Ear images is utilized work. 


\section{EAR-IIT Delhi}

This dataset version 1.0 mainly incorporates the ear Images collected from the graduates and Faculties at IIT Delhi, India. This entire dataset in the dataset is chiefly around ages grouped under $14-58$ years. The directory of 471 images is progressively counted for every user with unique identification number. The intention of these figures is about $272 \times 204$ pixels and reachable in jpeg format. This dataset endeavors the naturally normalized and cropped ear images of size about $50 \times 180$ pixels beside the authentic images. A further large adaption of ear dataset from 212 users with 754 ear images is incorporated.
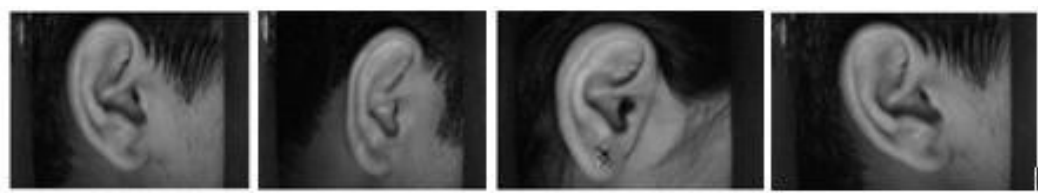

Figure 5. Illustration of Ear images from IIT Delhi Ear database Version 1.0.

\section{Experimental Results}

Originally these Ear figures are in gray scale format, it is very much accessible for filtering process. This filtering method includes Sobel filter which excludes the noise regions like thin hair, studs etc., and the Pre-processed process these figures are shown in the Figure 6.

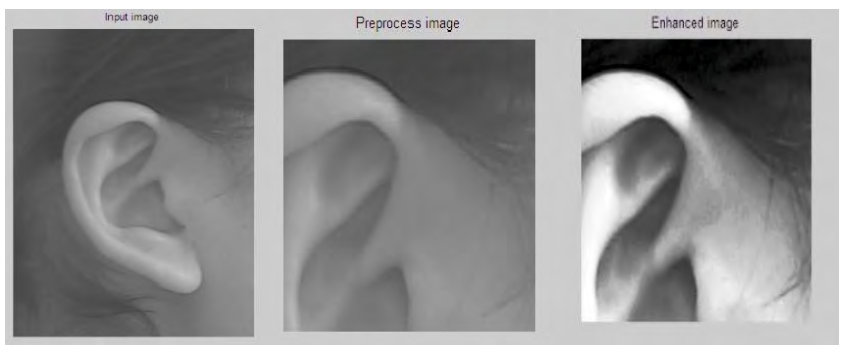

Figure 6. Results of Ear model (a) Input Figure (b) Preprocess Figure (c) Enhanced Image.

This Figure 6 shows the basic pre-processing and enhancement process which helps the enhanced image after histogram equalization that further moves to feature extraction of Gabor Filter. 


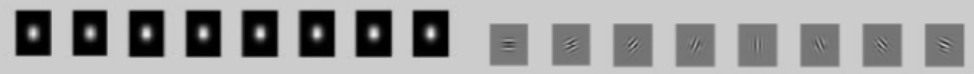

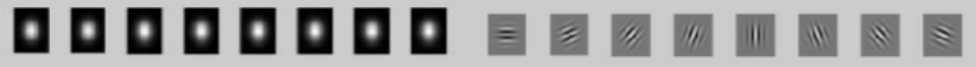

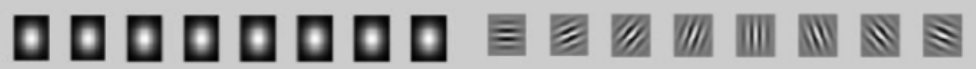

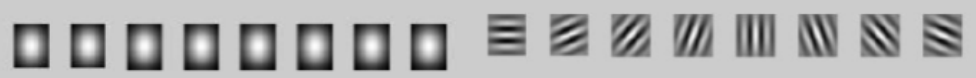

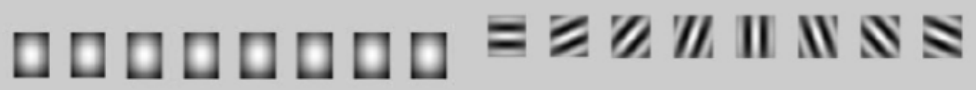

Figure 7. Magnitude and Real Parts of Gabor Filter.

The Figure 7 explains the Magnitude as well as Real Parts of Gabor feature Extraction which is determined from the Gabor Feature Extraction.

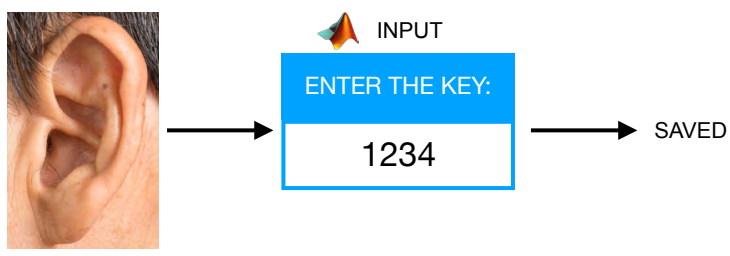

Figure 8. Encoding Process of Fuzzy Vault.

This Figure 8 explains the input image which are grouped as feature ve0ctors has been stored as [1234] in the Fuzzy Vault Database.

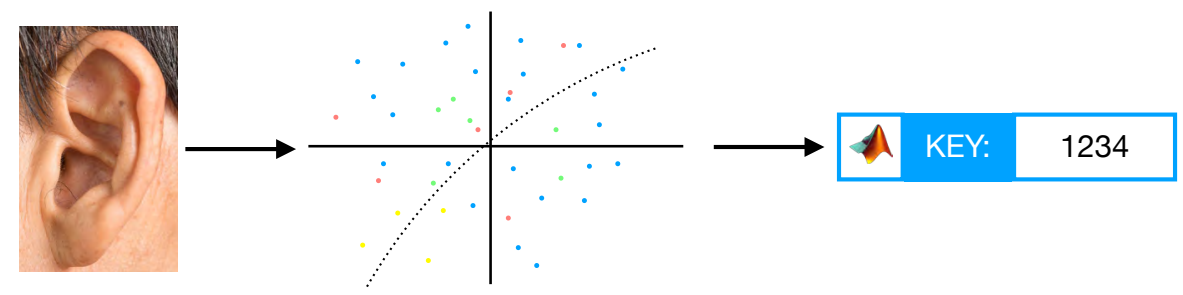

Figure 9. Decoding Process of Fuzzy Vault.

This Figure 9 shows the retrieval of Secret key from the Fuzzy Vault Database. It involves the grouped feature points that are indulged as chaff point's generation.

\section{Performance Evaluation Metrics}


To estimate this proposed biometric system it is established on Ear Images, several evaluation metrics are employed. This evaluation Metrics for this work involved are False Matching Rate (FMR), False Non-matching Rate (FNMR), Genuine Acceptance Rate (GAR) and Accuracy.

\section{False Matching Rate (FMR)}

This Matching Rate is based on the improper recognition of un-authorized People. The FMR is specified as resulted,

FMR = Number of un-authorized inputs with improper recognition

Total Number of Inputs

\section{False Non Matching Rate (FNMR)}

This Matching Rate is defined based on the improper recognition of authorized people. It has been defined as designed

FNMR $=$ Number of authorized Inputs that are falsely not recognized

Total Number of inputs

\section{Genuine Acceptance Rate (GAR)}

It is defined as the Probable of truly matching figures that are matched by the biometric security system with the entire images in the dataset.

\section{GAR=1-FNMR}

\section{Performance Analysis of this Proposed Work}

The results of this proposed image from Ear modalities are collected from 25 samples from various kinds of dataset. The results are taken based on the calculation of these evaluation metrics that is explained in Table 1.

Table 1. Analysis of biometric system with Ear modality.

\begin{tabular}{|c|c|c|c|c|}
\hline SI No. & FMR (\%) & FNMR (\%) & GAR (\%) & Accuracy (\%) \\
\hline 1 & 0.62 & 0.40 & 0.60 & 97.0 \\
\hline 2 & 0.60 & 0.40 & 0.61 & 98.0 \\
\hline 3 & 0.63 & 0.40 & 0.62 & 98.8 \\
\hline
\end{tabular}




\begin{tabular}{|c|c|c|c|c|}
\hline SI No. & FMR (\%) & FNMR (\%) & GAR (\%) & Accuracy (\%) \\
\hline 4 & 0.64 & 0.40 & 0.60 & 98.8 \\
\hline 5 & 0.72 & 0.30 & 0.70 & 96.0 \\
\hline 6 & 0.71 & 0.30 & 0.70 & 95.0 \\
\hline 7 & 0.70 & 0.30 & 0.70 & 98.1 \\
\hline 8 & 0.70 & 0.30 & 0.71 & 98.2 \\
\hline 9 & 0.80 & 0.20 & 0.80 & 96.0 \\
\hline 10 & 0.90 & 0.10 & 0.90 & 94.1 \\
\hline 11 & 0.70 & 0.30 & 0.70 & 98.8 \\
\hline 12 & 0.90 & 0.10 & 0.70 & 95.5 \\
\hline 13 & 0.60 & 0.40 & 0.60 & 94.1 \\
\hline 14 & 0.50 & 0.50 & 0.50 & 98.2 \\
\hline 15 & 0.70 & 0.30 & 0.70 & 98.8 \\
\hline 16 & 0.80 & 0.20 & 0.80 & 98.3 \\
\hline 17 & 0.60 & 0.40 & 0.60 & 94.0 \\
\hline 18 & 0.60 & 0.40 & 0.60 & 94.2 \\
\hline 19 & 0.70 & 0.30 & 0.70 & 95.5 \\
\hline 20 & 0.50 & 0.50 & 0.50 & 96.1 \\
\hline 21 & 0.40 & 0.60 & 0.40 & 97.1 \\
\hline 22 & 0.70 & 0.30 & 0.70 & 98.1 \\
\hline 23 & 0.80 & 0.20 & 0.80 & 98.9 \\
\hline 24 & 0.90 & 0.10 & 0.90 & 98.3 \\
\hline 25 & 0.50 & 0.50 & 0.50 & 98.4 \\
\hline
\end{tabular}

Table 1 shows the performance metrics of Ear modalities biometric system with various rates.

\section{CONCLUSION}

The stages in this work for this useful biometric system includes are (i) Pre-processing (ii) Gabor Feature Extraction (iii) Polynomial Construction of grouped vector from chaff points(iv)Identification and Authentication of secret key. These Proposed work biometric authentication systems with ear modalities are efficiently implemented in Matlab. Evaluation Metrics (FMR, FNMR, GAR) are calculated by frequently altering the key value. The analysis of this proposed work smoothened with better accuracy value as such $98.83 \%$. Further this idea will involve with multimodal biometric system to check its accuracy. 


\section{ACKNOWLEDGMENT}

We would like to be grateful for the International Research Centre of Kalasalingam Academy of Research and Education for investing financial assistance upon the scheme of University Research Fellowship (URF) and we also endorsed the Department of Electronics and Communication Engineering of Kalasalingam Academy of Research and Education, Tamil Nadu, India for providing usage of the computational facilities available in Signal Processing and VLSI Design laboratory that were set up with the assistance of the Department of Science and Technology (DST). 


\section{REFERENCES}

Anwar, A. S., Ghany, K. K. A., \& Elmahdy, H. (2015). Human ear recognition using geometrical features extraction. Procedia Computer Science, 65, 529-537. https://doi. org/10.1016/j.procs.2015.09.126

Arunachalam, M., \& Kanan, S. (2015). AES Based Multimodal Biometric Authentication using Cryptographic Level Fusion with Fingerprint and Finger Knuckle Print. The International Arab Fournal of Information Technology, 12(5), 431-440. https://www. semanticscholar.org/paper/AES-Based-Multimodal-Biometric-Authenticationusing-Arunachalam-Kannan/32a5f4d1d2f4c1d6ac521a486a7e20bc5c7b7da9

Bae, K., Noh, S., \& Kim, J. (2003). Iris feature extraction using independent component analysis. International Conference on Audio-and Video-Based Biometric Person Authentication. Springer, Berlin, Heidelberg. https://doi.org/10.1007/3-540-44887-X_97

Bansal, M., \& Hanmandlu, M. (2017). A new entropy function for feature extraction with the refined scores as a classifier for the unconstrained ear verification. Fournal of Electrical Systems and Information Technology, 4(1), 135-158. https://doi.org/10.1016/j. jesit.2016.10.006

Gandhimathi, A., \& Janarthanan, G. R. (2019). A Fuzzy vault based Multimodal biometric cryptosystem for enhancing security. International Fournal of Electrical and Computer Engineering, 768-773.

Gandhimathi, A., \& Radhamani, G. (2016). New chaff point based fuzzy vault for multimodal biometric cryptosystem using particle swarm optimization. Fournal of King Saud University-Computer and Information Sciences, 28, 381-394. https://core.ac.uk/ download/pdf/82045848.pdf

Hassaballah, M., Alshazly, H., \& Ali, A. A. (2019). Ear recognition using local binary patterns: A comparative experimental study. Expert Systems with Applications, 118, 182200. https://doi.org/10.1016/j.eswa.2018.10.007 
Hurley, D. J., Arbab-Zavar, B., \& Nixon, M. S. (2008). The ear as a biometric. In Jain A. K., Flynn P., Ross A. A. (eds) Handbook of Biometrics. Springer, Boston, MA, 131- 150. https://doi.org/10.1007/978-0-387-71041-9_7

Juels, A., \& Sudan, M. (2006). A fuzzy vault scheme. Designs, Codes and Cryptography, 38(2), 237-257.

Kacar, U., \& Kirci, M. (2018). ScoreNet: deep cascade score level fusion for unconstrained ear recognition. IET Biometrics, 8(2), 109-120. https://doi.org/10.1049/ietbmt.2018.5065

Koptyra, K., \& Ogiela, M. R. (2015). Fuzzy vault schemes in multi-secret digital steganography. In 2015 10th International Conference on Broadband and Wireless Computing, Communication and Applications (BWCCA). IEEE. https://doi.org/10.1109/ BWCAA.2015.87

Lakshmanan, L. (2013). Efficient person authentication based on multi-level fusion of ear scores. IET biometrics, 2(3), 97-106. https://doi.org/10.1049/iet-bmt.2012.0049

Lee, Y.J., Park, K. R., Lee, S. J., Bae, K., \& Kim, J. (2008). A new method for generating an invariant iris private key based on the fuzzy vault system. IEEE Transactions on Systems, Man, and Cybernetics, Part B (Cybernetics), 38(5), 1302- 1313. https://doi. org/10.1109/TSMCB.2008.927261

Nandakumar, K., Jain, A.K., \& Pankanti, S. (2007). Fingerprint-based fuzzy vault: Implementation and performance. IEEE transactions on information forensics and security, 2(4), 744-757. https://doi.org/10.1109/TIFS.2007.908165

Pflug, A., \& Busch, C. (2012). Ear biometrics: a survey of detection, feature extraction and recognition methods. IET biometrics, 1(2), 114-129. https://doi.org/10.1049/ietbmt.2011.0003

Rathgeb, C., Pflug, A., Wagner, J., \& Busch, G. (2016). Effects of image compression on ear biometrics. IET Biometrics, 5(3), 252-261. https://doi.org/10.1049/ietbmt.2015.0098 
Uludag, U., \& Jain, A. (2006). Securing fingerprint template: Fuzzy vault with helper data. In 2006 Conference on Computer Vision and Pattern Recognition Workshop (CVPRW'06). IEEE. https://doi.org/10.1109/CVPRW.2006.185

Vinothkanna, R., \& Amitabh, W. (2014). Fuzzy vault fusion based multimodal biometric human recognition system with fingerprint and ear. Fournal of theoretical and applied information technology, 59(2), 304-317. http://www.jatit.org/volumes/ Vol59No2/9Vol59No2.pdf

Yang, W., Sun, C., \& Wang, Z. (2011). Finger-knuckle-print recognition using Gabor feature and MMDA. Frontiers of Electrical and Electronic Engineering in China, 6(2), 374. https://doi.org/10.1007/s1 1460-011-0141-3

Yang, W., Sun, G., \& Zhang, L. (2011). A multi-manifold Discriminant analysis method for image feature extraction. Pattern Recognition, 44(8), 1649-1657. https://doi. org/10.1016/j.patcog.2011.01.019 
